\title{
Dexamethasone-induced cell death is restricted to specific molecular subgroups of multiple myeloma
}

\author{
Charlotte Kervoëlen ${ }^{1,2}$, Emmanuelle Ménoret ${ }^{1}$, Patricia Gomez-Bougie ${ }^{2,3}$, Régis \\ Bataille $^{2}$, Catherine Godon ${ }^{4}$, Séverine Marionneau-Lambot ${ }^{5}$, Philippe Moreau ${ }^{2,3}$, \\ Catherine Pellat-Deceunynck ${ }^{2,3}$, Martine Amiot ${ }^{2,3}$ \\ ${ }^{1}$ Myelomax, Nantes, France \\ ${ }^{2}$ CRCNA, University of Nantes, INSERM, UMR892, CNRS, UMR 6299, Nantes, France \\ ${ }^{3}$ Hematology Clinic, University Hospital, Nantes, France \\ ${ }^{4}$ Hematology Laboratory, University Hospital, Nantes, France \\ ${ }^{5}$ Plate-forme in vivo, Cancéropôle Grand Ouest, Nantes, France \\ Correspondence to: \\ Martine Amiot, e-mail: martine.amiot@inserm.fr \\ Keywords: multiple myeloma, glucocorticoid, glucocorticoid receptor, GILZ, Bim \\ Received: March 27, $2015 \quad$ Accepted: July 06, $2015 \quad$ Published: July 16, 2015
}

\section{ABSTRACT}

Due to its cytotoxic effect in lymphoid cells, dexamethasone is widely used in the treatment of multiple myeloma (MM). However, only a subset of myeloma patients responds to high-dose dexamethasone. Despite the undeniable anti-myeloma benefits of dexamethasone, significant adverse effects have been reported. We re-evaluate the anti-tumor effect of dexamethasone according to the molecular heterogeneity of MM. We demonstrated that the pro-death effect of dexamethasone is related to the genetic heterogeneity of MM because sensitive cell lines were restricted to MAF and MMSET signature subgroups, whereas all CCND1 cell lines $(n=10)$ were resistant to dexamethasone. We demonstrated that the glucocorticoid receptor expression was an important limiting factor for dexamethasone-induced cell death and we found a correlation between glucocorticoid receptor levels and the induction of glucocorticoidinduced leucine zipper (GILZ) under dexamethasone treatment. By silencing GILZ, we next demonstrated that GILZ is necessary for Dex induced apoptosis while triggering an imbalance between anti- and pro-apoptotic $\mathrm{Bcl}-2$ proteins. Finally, the heterogeneity of the dexamethasone response was further confirmed in vivo using myeloma xenograft models. Our findings suggested that the effect of dexamethasone should be re-evaluated within molecular subgroups of myeloma patients to improve its efficacy and reduce its adverse effects.

\section{INTRODUCTION}

Multiple myeloma (MM) is an incurable plasma cell malignancy despite considerable improvements to survival due to the introduction of new drugs, such as proteasome inhibitors and immunomodulatory agents [1]. Glucocorticoids (GCs) are widely used in the treatment of MM, mainly in combination regimens. Among GCs, dexamethasone (Dex) is used in all phases of treatment, including induction, consolidation and maintenance. In 1992, Alexanian et al showed that high doses of Dex were effective in about half of untreated MM patients and that the efficacy of the combination of vincristine, doxorubicin and dexamethasone was mainly due to the high doses of Dex [2]. Following the introduction of thalidomide, Dex has mainly been used in combination regimens because the combination of Dex with thalidomide demonstrates significantly superior response rates in newly diagnosed MM patients compared with Dex alone [3]. More recently, Dex associated with bortezomib and lenalidomide has appeared as the most promising drug association recommended for high-risk patients [4].

The effects of GCs occur through GC binding to the GC receptor GR, a member of the type I nuclear receptor superfamily. GR is transcribed from a single 
gene, NR3C1, located on chromosome 5. However, GR actions are dependent on multiple receptor isoforms. Alternative splicing of GR pre-mRNA produces five distinct GR protein isoforms: the predominant full-length GR $\alpha$, the dominant negative GR $\beta$, and the three less-well characterized and less abundant isoforms GR $\gamma$, GR-A and GR-P [5, 6]. Resistance to GCs is not fully understood because multiple molecular mechanisms are involved and are likely cell-type specific [7, 8]. However, altered or low-level GR expression accounts for inherent resistance to GCs [8]. Resistance may also occur downstream of GR. GCs bind GR, which then translocates to the nucleus and interacts with either GC-response elements to induce gene transcription (transactivation) or directly interacts with transcription factors, such as NFKB or AP-1 to repress their activity (transrepression). The transactivation activity of GR is highly regulated and required for GCinduced apoptosis involving the intrinsic mitochondrial pathway $[6,9]$. The TSC22D3 gene encoding the GCinduced leucine zipper protein GILZ is one of the most strongly up-regulated genes by GCs [10]. An important overlap between the effect of GILZ and those of GCs was demonstrated, suggesting that GILZ is a critical mediator of the therapeutic effect of GCs [11-13]. GILZ protein is involved in numerous protein/protein interactions and thus regulates multiple signaling pathways, including $N F-\kappa B$ and Ras [11-13].

Among the multiple candidate genes involved in GC-induced apoptosis, BCL2L11, which encodes the proapoptotic Bim protein, appears to be the most important. In 2003, BCL2L11 was identified as a GC-induced death gene (18). Since that time, numerous studies have shown that Bim is a key mediator of GC-induced cell death in lymphoid cells [9, 14-16].

$\mathrm{MM}$ is molecularly heterogeneous, with chromosomal abnormalities that include full or partial deletions of chromosomes 13 or 17, amplification of $1 \mathrm{q} 21$, recurrent translocations of $14 \mathrm{q} 32$ or hyperdiploidy [17-19]. The recurrent 14q32 translocation and hyperdiploidy are associated with distinct gene expression profiles that define several groups, namely the HY, MS, CD-1/2 and MF groups, characterized by the hyperdiploid, MMSET, CCND1 and MAF signatures, respectively. The MS and MF subgroups have been associated with poor overall survival $[20,21]$. Although c-maf translocation is only found in 5 to $10 \%$ of patients, c-maf is overexpressed in approximately $50 \%$ of patients and is regulated both by the MEK and MMSET pathways [22, 23].

Because only $50 \%$ of untreated MM patients respond to high doses of Dex [2], our study was undertaken to re-evaluate the anti-tumor effect of Dex according to the molecular heterogeneity of MM patients using a large collection of myeloma cell lines $(n=31)$ that are representative of the molecular translocations found in patients.

\section{RESULTS}

\section{The cellular response to Dex was correlated with the molecular subtype of human myeloma cell lines (HMCLs)}

Although it is well accepted that Dex induces apoptosis in MM, the variability of the response among HMCLs with different genetic backgrounds harboring the main recurrent translocations has never been investigated. HMCLs were classified into MF, MS and CCND1 subgroups according to their IgH translocation (Supplementary Table S1). All HMCLs that did not present the main recurrent translocations leading to $M A F$, MMSET or CCND1 over-expression were classified into the "Others" subgroup. Cell death induction was assessed across a panel of 31 HMCLs following Dex treatment for 72 hours. The dose of $1 \mu \mathrm{M}$ of Dex was chosen because there is no dose response effect in cell death induction for Dex concentrations ranging from 0.1 to $10 \mu \mathrm{M}$ (Supplementary Figure S1A). The ability of Dex to induce myeloma cell death was very heterogeneous within the different subgroups of HMCLs (Figure 1A, Supplementary Table S1). Dex-induced apoptosis was restricted to the MF and MS subgroups, with a mean number of apoptotic cells of $25 \%$ and $24 \%$, respectively (Figure 1A). In contrast, no cell death was induced by Dex in any of the CCND1 HMCLs $(n=10)$. We first determined whether cell death was associated with caspase activation and PARP1 cleavage in a selected panel of cell lines, including Dexsensitive ( $>15 \%$ cell death) and Dex-resistant HMCLs, which were representative of the molecular diversity of MM (Figure 1B). The results in Figure 1C showed that the activation of caspase 3 and the PARP1 cleavage were effective in all sensitive cell lines. Then, the effect of Dex was assessed in 19 primary myeloma cells, showing that cell death induction was highly heterogeneous from 0 to 96\% (Table 1, Supplementary Figure S1B). Four samples harbored an $\operatorname{IgH}$ translation, one with a $\mathrm{t}(14 ; 16)$, one with a $\mathrm{t}(4 ; 14)$ and two with a $\mathrm{t}(11 ; 14)$. Of note, the MF and MS patient were sensitive $(92 \%$ and $49 \%$ cell death respectively), whereas the CCND1 patients were resistant ( $2 \%$ and $4 \%$ cell death). Two samples at diagnosis appeared resistant to Dex, indicating that primary tumor cells could present an inherent resistance to Dex.

\section{High NR3C1 expression characterized the MF and hyperdiploid (HY) subgroups of myeloma patients}

Because Dex exerts its action through the GR, we investigated GR expression by transcriptomic Affymetrix analysis of the NR3Cl gene in the different HMCL subgroups. $N R 3 C 1$ gene expression was heterogeneous among the HMCL subgroups ( $p=0.02)$; however, the MF and MS subgroups exhibited a higher expression 
Table 1: Dex sensitivity and characteristics of primary myeloma cells

\begin{tabular}{|c|c|c|c|c|c|c|c|}
\hline \multicolumn{4}{|c|}{ Patient characteristics } & \multirow{2}{*}{$\frac{\text { Dex sensitivity }}{\% \text { cell death }(1 \mu \mathrm{M})}$} & \multicolumn{3}{|c|}{ FISH analysis } \\
\hline Sample & Disease & Status & Origin & & $\mathrm{t}(4 ; 14)$ & t $(14 ; 16)$ & $\mathrm{t}(11 ; 14)$ \\
\hline $1 *$ & MM & $\mathrm{R}$ & $\mathrm{BM}$ & 96 & - & - & - \\
\hline 2 & $\mathrm{pPCL}$ & $\mathrm{D}$ & PB & 92 & - & + & - \\
\hline $3 *$ & MM & $\mathrm{R}$ & $\mathrm{BM}$ & 87 & - & - & - \\
\hline 4 & $\mathrm{pPCL}$ & $\mathrm{D}$ & PB & 84 & - & - & - \\
\hline 5 & MM & $\mathrm{R}$ & $\mathrm{BM}$ & 60 & - & - & - \\
\hline 6 & MM & $\mathrm{R}$ & $\mathrm{BM}$ & 58 & - & - & - \\
\hline 7 & MM & $\mathrm{D}$ & $\mathrm{BM}$ & 49 & + & - & - \\
\hline $8 *$ & MM & $\mathrm{D}$ & $\mathrm{BM}$ & 33 & - & - & - \\
\hline 9 & $\mathrm{pPCL}$ & $\mathrm{D}$ & $\mathrm{PB}$ & 29 & - & - & - \\
\hline 10 & $\mathrm{pPCL}$ & $\mathrm{D}$ & PB & 26 & - & - & - \\
\hline 11 & $\mathrm{pPCL}$ & $\mathrm{D}$ & PB & 20 & - & - & - \\
\hline 12 & sPCL & $\mathrm{R}$ & PB & 17 & - & - & - \\
\hline $13^{*}$ & MM & $\mathrm{R}$ & $\mathrm{BM}$ & 8 & - & - & - \\
\hline 14 & MM & $\mathrm{R}$ & BM & 8 & - & - & - \\
\hline 15 & MM & $\mathrm{R}$ & BM & 4 & - & - & + \\
\hline 16 & sPCL & $\mathrm{R}$ & PB & 2 & - & - & + \\
\hline 17 & sPCL & $\mathrm{R}$ & $\mathrm{PB}$ & 0 & - & - & - \\
\hline $18 *$ & MM & D & $\mathrm{BM}$ & 0 & - & - & - \\
\hline 19 & pPCL & D & $\mathrm{PE}$ & 0 & - & - & - \\
\hline
\end{tabular}

Abbreviations: MM, multiple myeloma; pPCL, primary plasma cell leukemia; sPCL, secondary plasma cell leukemia; D, diagnosis; R, relapse; $\mathrm{BM}$, bone marrow; $\mathrm{PB}$, peripheral blood; PE, pleural effusion.

Plasma cells were purified $(*)$ or not with CD138-immunomagnetic beads depending on the myeloma infiltration and the total number of cells. Cell death was assessed after 48 hours Dex treatment by the loss of CD138 staining.

compared with the CCND1 and Others subgroups (1.8fold mean increase, $p=0.002$ ) (Figure 2A). We also found that $N R 3 C 1$ expression was significantly higher in the $\mathrm{MF}$ subgroup compared with all other HMCLs (1.4-fold mean increase, $p=0.029$ ) (Figure 2A). We then took advantage of the publicly available Affymetrix gene expression microarray data from 309 newly diagnosed MM patients to analyze $\mathrm{NR} 3 \mathrm{Cl}$ expression in the MF, MS, CCND1 and HY groups of patients, as previously defined [18]. We first noticed that none of the patients completely lacked NR3C1 expression but that NR3C1 levels were significantly different between the molecular subgroups $(p<0.0001)$. Of note, MF patients expressed significantly higher levels of NR3C1 than all other subgroups (1.8fold mean change, $p<0.0001$ ) (Figure 2B), suggesting that the specific regulation of $N R 3 C 1$ expression may occur in MF patients. Moreover, HY patients expressed significantly higher levels of $N R 3 C 1$ than MS and CCND1 patients (1.3-fold mean increase, $p<0.0001)$ (Figure 2B), which may be explained by the localization of the $N R 3 C 1$ gene on chromosome 5. Indeed, chromosome 5 trisomy is frequent in hyperdiploid MM [24].

\section{Levels of GR expression appear to be a limiting factor for Dex-induced cell death}

To deepen the understanding of Dex sensitivity, we established two Dex-resistant cell lines, NAN8DR and MM.1SDR, obtained by long-term culture of the parental cell lines with a low Dex concentration (Supplementary Figure S2). NAN8DR and MM.1SDR cells were characterized by an absence of cell death induction $(0 \%$ cell death in both NAN8DR and MM.1SDR compared with $58 \%$ and $35 \%$ in the parental cell lines, respectively) associated with nearly no caspase activation and no PARP1 cleavage under Dex treatment (Supplementary Figure S2). In contrast, their sensitivity to bortezomib or lenalidomide was unchanged (Supplementary Figure S2). To determine whether long-term Dex treatment affected the GR levels in resistant cell lines compared with their respective parental 
A

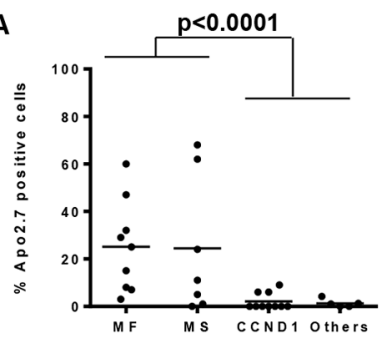

B

\begin{tabular}{cc} 
HMCL & Translocation \\
\hline BCN & $\mathrm{t}(14 ; 16)$ \\
MM.1S & $\mathrm{t}(14 ; 16)$ \\
L363 & $\mathrm{t}(20 ; 22)$ \\
XG-6 & $\mathrm{t}(16 ; 22)$ \\
OPM-2 & $\mathrm{t}(4.14)$ \\
NAN-8 & $\mathrm{t}(4 ; 14)$ \\
U266 & $\mathrm{t}(11 ; 14)$ \\
KMS12-PE & $\mathrm{t}(11 ; 14)$
\end{tabular}

\begin{tabular}{l} 
Molecular \\
subgroup \\
\hline MF \\
MF \\
MF \\
MF \\
MS \\
MS \\
CCND1 \\
CCND1
\end{tabular}

$\%$ cell death

$60 \pm 8$

$47 \pm 2$

$29 \pm 3$

$3 \pm 2$

$68 \pm 4$

$62 \pm 3$

$6 \pm 2$

$0 \pm 0$
Dex Sensitivity

Sensitive

Sensitive

Sensitive

Resistant

Sensitive

Sensitive

Resistant

Resistant

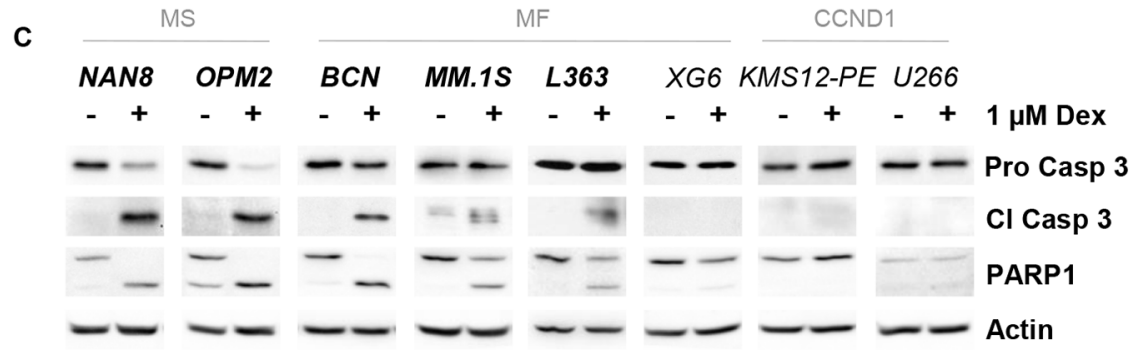

Figure 1: Dex sensitivity is heterogeneous and restricted to the MS and MF HMCL subgroups. A. HMCLs $(n=31)$ were treated with $1 \mu \mathrm{M}$ Dex for 72 hours, and cell death was assessed by Apo2.7 staining. HMCLs were classified into 4 groups as follow: MS, MF, CCND1 and Others for no or no recurrent translocation cell lines. Of note, HMCLs harboring a $t(14 ; 20), t(20 ; 22)$ or $t(16 ; 22)$ were included in the MF subgroup. Each symbol represents one HMCL. Statistical analysis was performed using the Mann Whitney test. B. Characteristics of selected sensitive and resistant cell lines. Dex sensitivity was defined as follows: sensitive, $\geq 15 \%$ apoptotic HMCL cells and resistant, $<15 \%$ apoptotic HMCL cells. C. Cell death signature was analyzed by immunoblotting after 24 hours of Dex treatment. Actin was used as a loading control.

cell lines, we assessed NR3C1 levels by Q-PCR. NAN8DR and MM.1SDR cells expressed very low NR3C1 levels compared with the parental cell lines (Figure 2C). We next compared the NR3C1 levels in our selected panel of cell lines and showed that sensitive cell lines demonstrated higher NR3C1 levels than resistant cell lines $(p=0.036)$ (Figure 2D). However, we noticed that among the resistant cell lines, KMS12-PE expressed higher NR3C1 levels than other resistant cell lines. Additionally, with some exceptions, we showed a correlation between NR3C1 mRNA expression and the pro-apoptotic effect of Dex (Figure 2E, $p<0.0001$, Rho: 0.70). Finally, we found a correlation between $N R 3 C 1$ and $M A F$ expression suggesting that $N R 3 C 1$ expression may be linked with $M A F$ expression (Figure 2F, $p=0.0012$, Rho $=0.55)$. In its inactive form, GR is located in the cytoplasm; once activated by GC, it is translocated to the nucleus [25]. Thus, we analyzed GR protein expression in the cytosolic fraction and its translocation to the nucleus. Dex treatment induces the total translocation of GR to the nuclear fraction irrespective of its endogenous level (Figure 2G). Altogether, these results suggested that low levels of GR appear to be one of the first limiting events in Dex resistance.

\section{The strong up-regulation of GILZ was necessary for Dex-induced apoptosis}

Using the same panel of HMCLs, we further investigated the expression of GILZ, a protein encoded by the TSC22D3 GC-transactivated gene. After Dex exposure, TSC22D3 levels were up-regulated in most cell lines (Figure 3A). However, resistant cell lines failed to upregulate $T S C 22 D 3$ to the same extent as sensitive cell lines (Figure 3A). Of note, a correlation was found between the levels of GR expression and Dex-induced TSC22D3 upregulation ( $p=0.03$, Rho: 0.68 ) (Figure $3 \mathrm{~B}$ ). Analysis of the GILZ protein under Dex treatment confirmed a stronger up-regulation of GILZ not only in sensitive cell lines but also in KMS12-PE cells, which expressed high levels of GR (Figure 3C). To assess whether GILZ may influence the Dex response, we next performed transient knockdown of GILZ in both OPM2 and BCN cell lines. Indeed, GILZ silencing strongly reduced Dex-induced apoptosis (54\% decrease, $p=0.008)$ in OPM2 cell line (Figure 3D) and to a lesser extent in $\mathrm{BCN}(30 \%$ decrease, $p=0.03)$ (Supplementary Figure S3). However, this weaker effect on Dex-induced apoptosis in BCN compared to OPM2 is probably due to the less efficient GILZ silencing (40\% versus 83\% inhibition respectively). Altogether, these results highlighted the implication of GILZ in Dex-induced apoptosis.

\section{GILZ was involved in Bim up-regulation and Bcl- $x_{L}$ down-regulation associated with Dex- induced apoptosis in HMCLs}

Among the pro-apoptotic Bcl-2 family proteins, Bim, a BH3-only member, has frequently been identified 

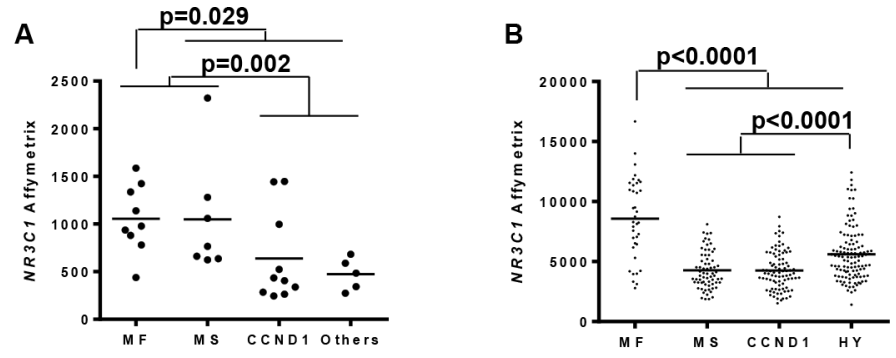

C

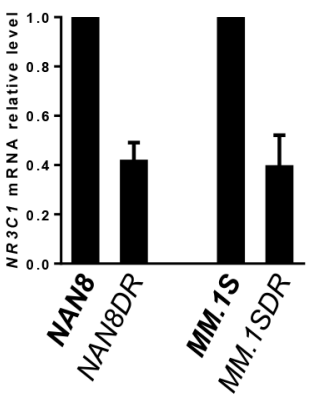

D

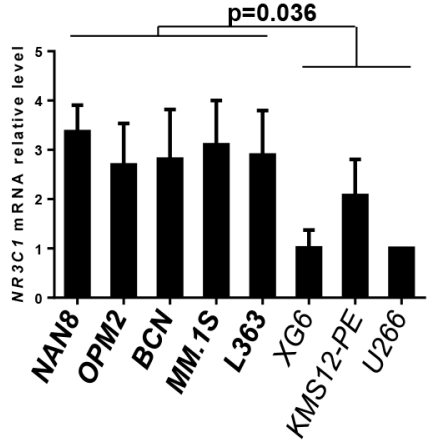

E

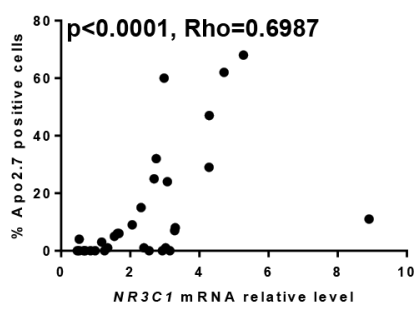

$\mathbf{F}$

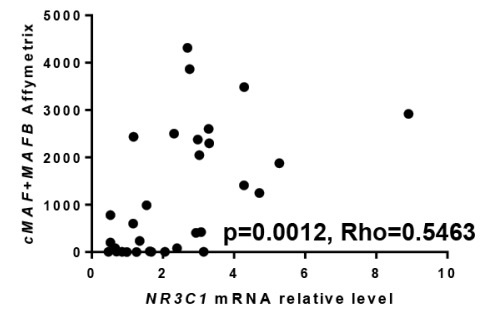

$\mathbf{G}$

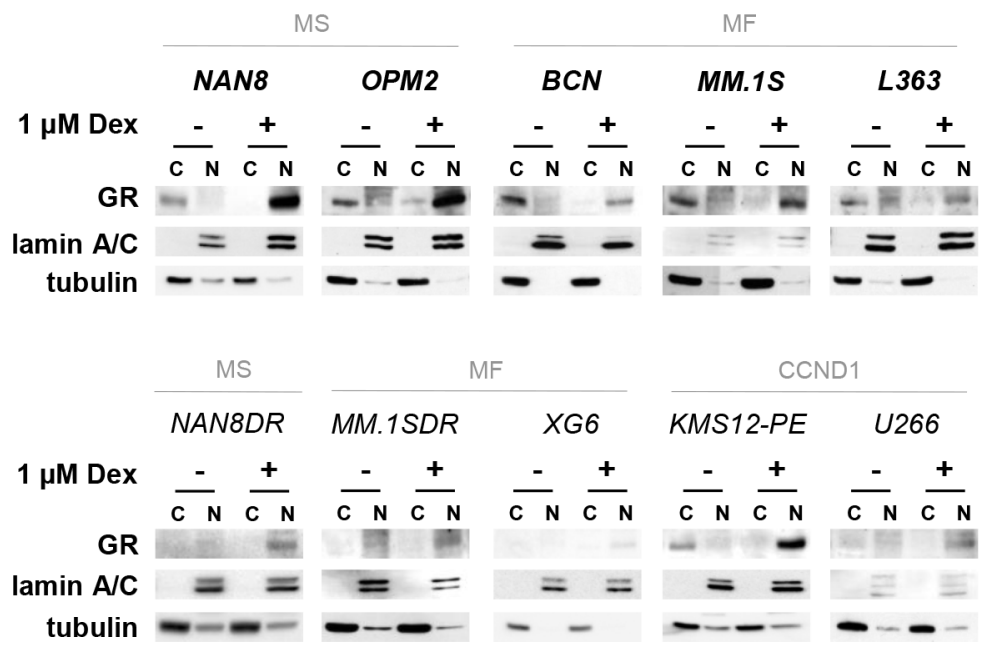

Figure 2: Analysis of both $\mathrm{NR} 3 \mathrm{C} 1$ gene and GR protein expression in myeloma subgroups. A, B. $N R 3 C 1$ gene expression of HMCLs and 309 newly diagnosed MM patients was assessed by transcriptomic Affymetrix analysis and plotted for the respective HMCL subgroups (A), or for the MF, MS, CCND1 and hyperdiploid (HY) patient groups (B) Each symbol represents one HMCL or one patient. Statistical analysis was performed using the Mann Whitney test. C, D. NR3C1 mRNA expression of Dex-resistant cell lines (C) and HMCL collection (D) was assessed by Q-PCR. NR3C1 mRNA expression of generated Dex-resistant cell lines was normalized to NR3C1 mRNA expression of the parental cell lines. NR3C1 mRNA expression of HMCLs was normalized to NR3C1 mRNA expression of U266 cell line. The mean \pm SD of 3 experiments is presented. Sensitive cell lines are indicated by thick characters, and resistant cell lines are indicated by thin characters. Statistical analysis was performed using the Mann Whitney test. E, F. The relative NR3C1 mRNA level was analyzed versus the percentage of Apo2.7-positive cells under Dex treatment (E) or versus MAF Affymetrix expression (F). MAF gene expression was assessed by addition of the $c M A F$ and $M A F B$ values of transcriptomic Affymetrix expression. Spearman's rank correlation coefficients are indicated. G. After 24 hours of Dex treatment, the translocation of GR was assessed by analyzing the expression of GR in both the nuclear $(\mathrm{N})$ and cytosolic $(\mathrm{C})$ fractions. Each fraction $(20 \mu \mathrm{g})$ was subjected to western blot analysis for GR expression. Lamin A/C and tubulin were included as fraction purity markers. Sensitive cell lines are indicated as above. 
A

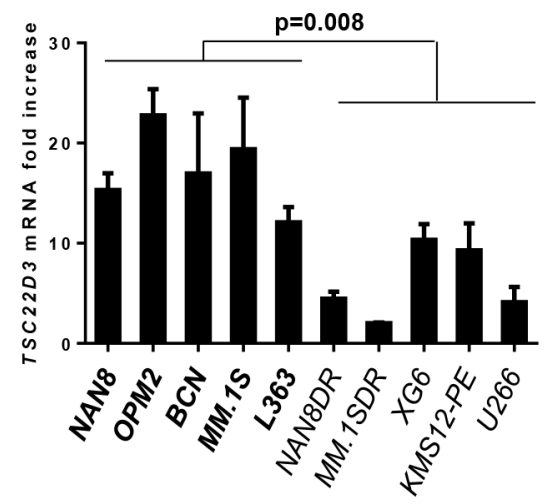

B

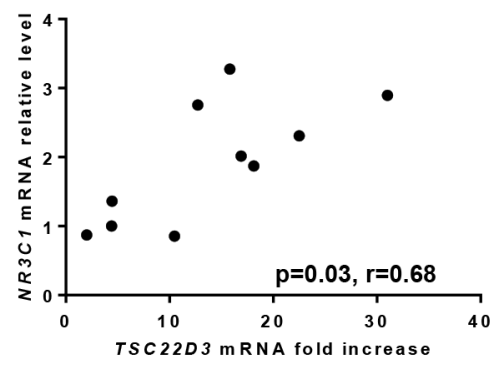

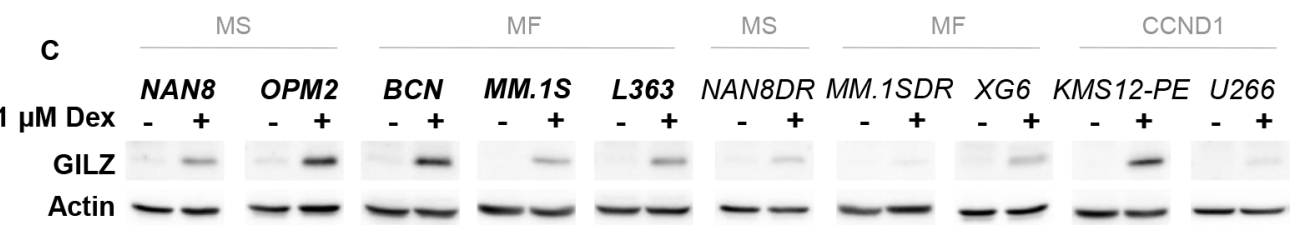

D

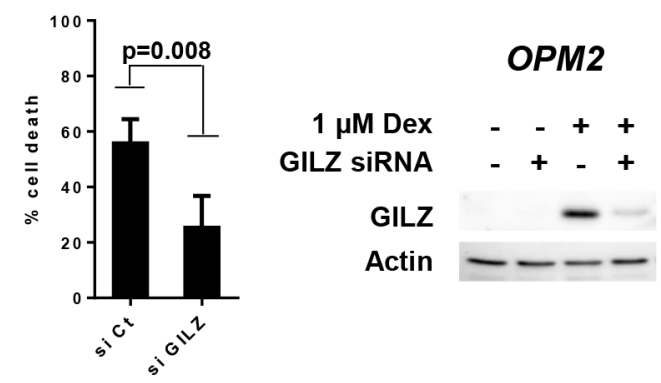

Figure 3: GILZ up-regulation is necessary for Dex-induced apoptosis. A. TSC22D3 mRNA expression was assessed by Q-PCR after 24 hours of Dex treatment. TSC22D3 mRNA induction was assessed by calculating the fold increase relative to the untreated cell line. The mean \pm SD of 3 experiments is presented. Statistical analysis was performed using the Mann Whitney test. B. Analysis of the relative $N R 3 C 1$ mRNA level versus the fold increase of TSC22D3 mRNA under Dex treatment. Spearman's rank correlation coefficient is indicated. C. Cell lines were treated with Dex for 24 hours and cell lysates were immunoblotted with a GILZ antibody. D. Following GILZ silencing, OPM2 cells were treated with Dex. Cell death was assessed by Apo2.7 staining after 48 hours of Dex treatment. Data represent the mean \pm SD of 4 independent experiments. Statistical analysis was performed using Wilcoxon matched-pairs signed rank test. After 16 hours of Dex treatment, GILZ silencing was analyzed by immunoblotting.

as one of the key mediators of GC-induced apoptosis [14-16]. In addition to Bim, Puma another BH3-only member was also shown to contribute to GC-induced apoptosis [15]. Furthermore, based on a previous microarray analysis in MM.1S cells [26], the BCL2L1 gene that encodes Bcl- $\mathrm{x}_{\mathrm{L}}$ was shown to be the only anti-apoptotic gene strongly down-regulated by Dex. We demonstrated that a strong up-regulation of all Bim isoforms occurred mainly in sensitive cell lines but also in KMS12-PE Dexresistant cells (Figure 4A). When Puma was endogenously expressed in the HMCLs, we observed a weak Puma upregulation in parallel to Bim up-regulation (Figure 4A). The anti-apoptotic Mcl-1 protein was not modulated with the exception of OPM2 where Mcl-1 is down-regulated. In contrast to $\mathrm{Mcl}-1$, we showed that $\mathrm{Bcl}-\mathrm{x}_{\mathrm{L}}$ protein expression was strongly decreased in all Dex-sensitive HMCLs, whereas its expression was weakly modified in
Dex-resistant HMCLs (Figure 4A). These results may indicate that the apoptotic response is associated with an imbalance between pro- and anti-apoptotic proteins. We next demonstrated that Bim silencing in OPM2 cells caused a significant decrease in Dex-induced cell death $(51 \%, p=0.004)$ (Figure 4B). Finally, because the resistant KMS12-PE HMCL is characterized by an increase in Bim under Dex treatment but an absence of Bcl- $\mathrm{x}_{\mathrm{L}}$ downregulation, we silenced $\mathrm{Bcl}-\mathrm{x}_{\mathrm{L}}$ to determine whether its down-regulation could overcome Dex resistance. Despite the complete silencing of Bcl- $\mathrm{x}_{\mathrm{L}}$ in KMS12-PE cells, Dex did not induce significant cell death, indicating that Bcl- $x_{L}$ down-regulation concomitant to Bim up-regulation was not sufficient to counteract Dex resistance in this CCND1 HMCL (Figure 4C). Of interest, the silencing of GILZ impairs both Bim up-regulation and Bcl- $\mathrm{x}_{\mathrm{L}}$ down-regulation under Dex treatment (Figure 4D). Finally, 


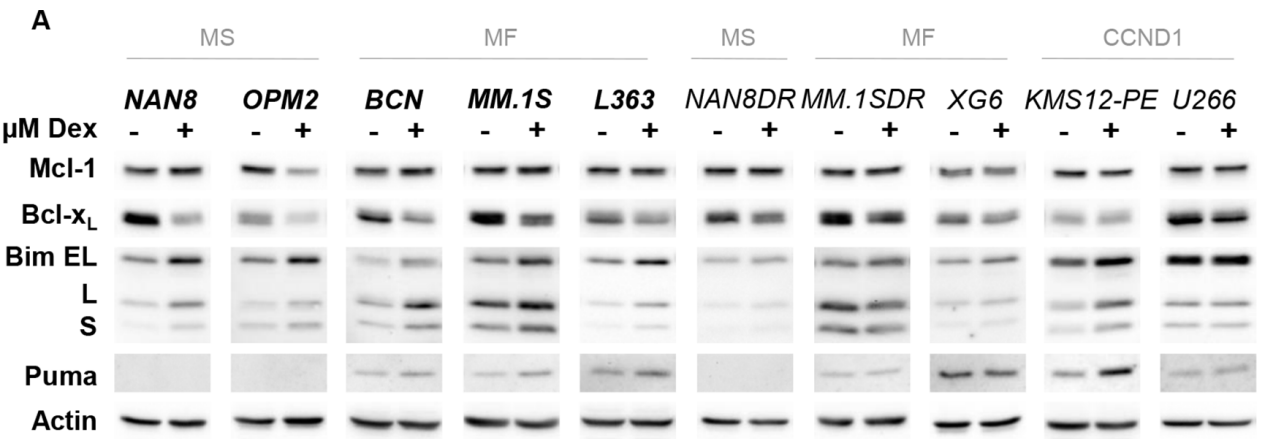

B

OPM2

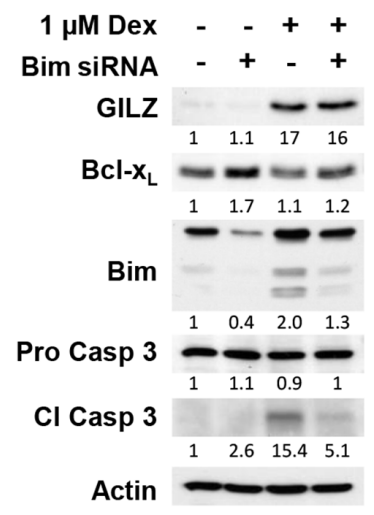

D

\section{OPM2}

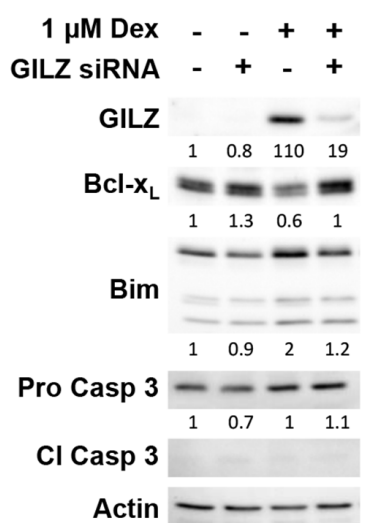

C

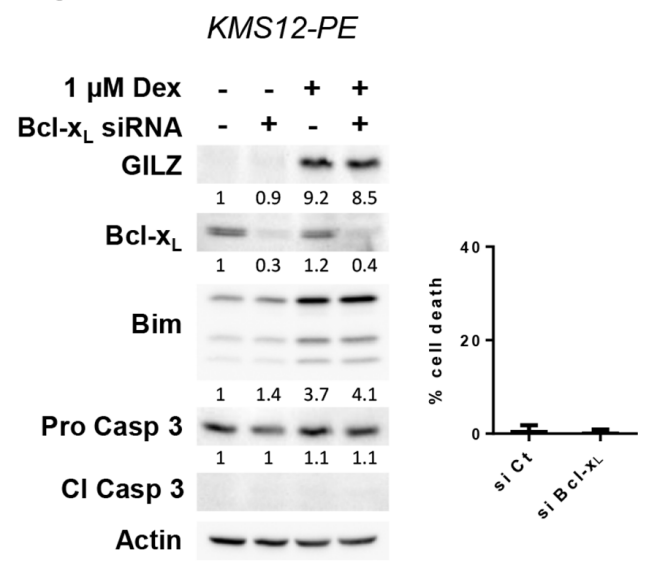

$E$

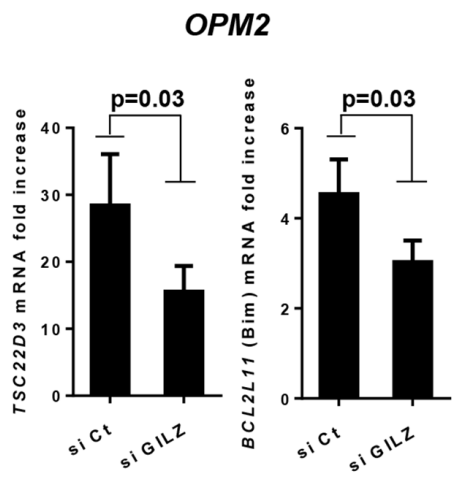

Figure 4: GILZ is involved in Bim up-regulation and Bcl- $x_{L}$ down-regulation associated with Dex-induced apoptosis. A. Cell lines were treated with Dex for 24 hours and lysates were immunoblotted with Mcl-1, Bcl- $\mathrm{x}_{\mathrm{L}}$, Bim and Puma antibodies. B. Following siRNA transfection, OPM2 cells were treated with Dex for 48 hours. The expression of Bim, Bcl- $\mathrm{x}_{\mathrm{L}}$, GILZ and caspase 3 were analyzed by immunoblotting and normalized (arbitrary unit) to actin expression. Apoptosis was quantified by Apo2.7 staining. The results show the mean \pm SD of 4 independent experiments. Statistical analysis was performed using Wilcoxon matched-pairs signed rank test. C. Following siRNA transfection, KMS12-PE cells were treated with Dex for 48 hours. The protein expressions were analyzed by immunoblotting and normalized (arbitrary unit) using actin expression. Apoptosis was quantified by Apo2.7 staining. The results show the mean \pm SD of 3 independent experiments. D. GILZ was transiently silenced in OPM2 cells for 48 hours, before to be treated with Dex for 16 hours. The protein expressions were analyzed by immunoblotting and normalized (arbitrary unit) to actin expression. E. Following GILZ silencing, OPM2 cells were treated with Dex for 8 hours. TSC22D 3 and BCL2L11 mRNA induction was assessed by calculating the fold increase relative to the untreated cell line. The mean \pm SD of 5 independent experiments is presented. Statistical analysis was performed using Wilcoxon matched-pairs signed rank test. 
we demonstrated that GILZ silencing decreased BCL2L11 (Bim) up-regulation at the transcriptional level $(33 \%$ decrease, $p=0.03$ ) (Figure 4E). Altogether, these results seem to indicate a pivotal role for GILZ in Dex-induced cell death through the regulation of the $\mathrm{Bcl}-2$ protein network.

\section{Dexamethasone reduced tumor cell growth of in vitro sensitive but not resistant cells}

To assess whether the differential in vitro activity of Dex could reflect its in vivo activity, both Dex-sensitive (OPM2) and Dex-resistant (KMS12-PE) cell lines were xenografted into SCID mice. Mice were subcutaneously inoculated with $12 \times 10^{6}$ cells. When mice bore tumors of similar sizes, they were randomly separated into 2 groups and received either vehicle (control) or Dex $(1 \mathrm{mg} / \mathrm{kg}$ ) 5 days a week for 3 consecutive weeks (Figure 5). When the tumor sizes exceeded $2000 \mathrm{~mm}^{3}$, mice were sacrificed on day 19 and 17 for mice grafted with OPM2 and KMS12PE cells respectively. Dex significantly inhibited OPM2 tumor growth (Figure 5A) $(p<0.05)$, but failed to reduce tumor growth of the in vitro resistant KMS12-PE cells (Figure 5B). Taken together, these data demonstrated that the in vivo pro-apoptotic Dex effect in pre-clinical myeloma models is related to in vitro Dex-induced cell death.

\section{DISCUSSION}

Because clinical practice has frequently been based on empirical studies, our study was undertaken to re-evaluate Dex, a conventional therapy, according to the molecular classification of MM patients. Our results highlighted that the Dex pro-apoptotic effect was restricted to the MF and MS subgroups of HMCLs, whereas all other cell lines were totally resistant to Dex. In particular, all CCND1 HMCLs and primary samples were resistant to Dex-induced cell death. The analysis of Dex sensitivity in relation to $N R 3 C 1$ levels revealed that the MF subgroup expressed the highest levels of $N R 3 C 1$, which may partially explain their stronger sensitivity to Dex. A previous study has also reported a link between GC and MAF showing that GC reduced c-maf protein levels via an ubiquitination-dependent degradation [27]. Nevertheless, more investigations will be necessary to explain the overexpression of $\mathrm{NR} 3 \mathrm{Cl}$ in the MF subgroup. In addition, the localization of the NR3C1 gene on chromosome 5 probably explains the higher levels of $N R 3 C 1$ found in the $\mathrm{HY}$ subgroup compared with the MS and CCND1 subgroups because chromosome 5 trisomy is frequently found in hyperdiploid MM [24]. The generation of MF or MS Dexresistant HMCLs after long-term exposure to Dex led to an important decrease in $N R 3 C 1$, indicating that an $N R 3 C 1$ level seems necessary for Dex-induced apoptosis. To support this hypothesis, we showed a correlation between NR3C1 levels and the apoptotic response in HMCLs. Consistently, a poor prognosis has been associated with low $N R 3 C 1$ expression in MM patients [28, 29]. In addition, we demonstrated that Dex induced the total translocation of GR to the nucleus in all HMCLs tested, even in resistant cell lines, showing that the ability of GR to translocate to the nucleus is not a limiting step in Dex resistance.

Many studies in hematopoietic cells, essentially in $\mathrm{T}$ and dendritic cells, have identified GILZ as one of the main GC-regulated genes [30, 31]. The GILZ promoter includes 6 GC-responsive element motifs that allow for GR
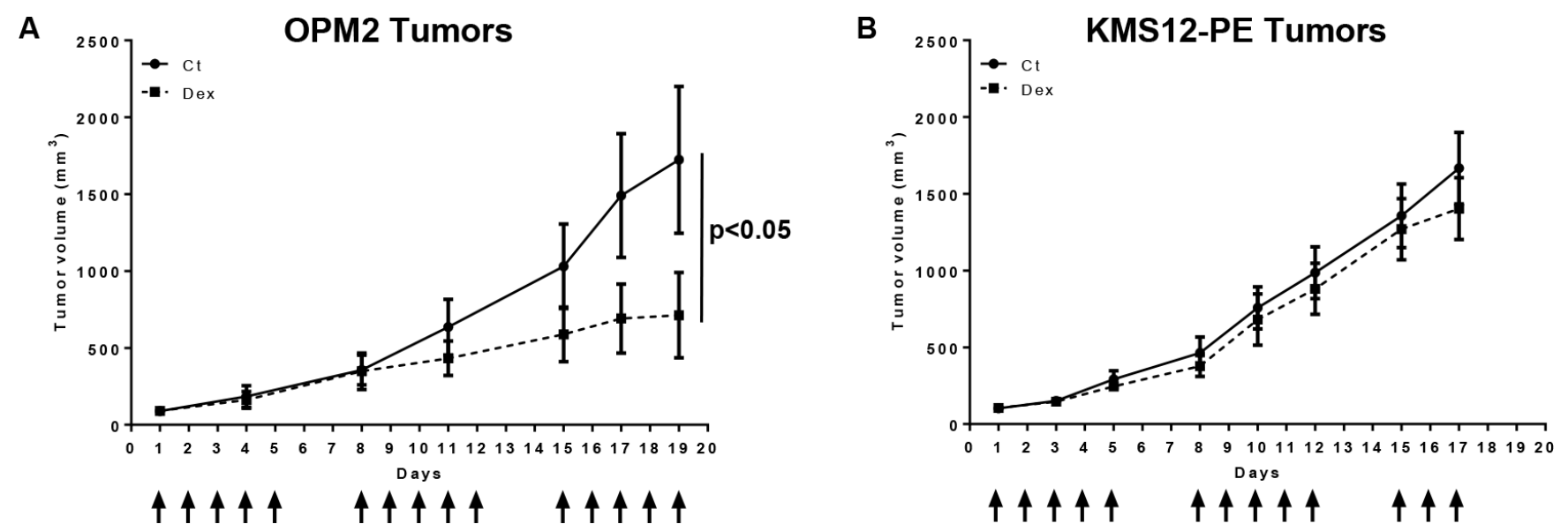

Figure 5: Dexamethasone reduces tumor cell growth of in vitro sensitive OPM2 but not resistant KMS12-PE cells in xenograft models. A. Dex treatment inhibited in vivo OPM2 tumor growth. Mice were subcutaneously injected with $12 \times 10^{6}$ OPM 2 cells. The mice received either vehicle $(\mathrm{Ct})$ or Dex $(1 \mathrm{mg} / \mathrm{kg}$, i.p.) 5 days per week for 3 consecutive weeks. Arrows indicate injections. The mice were then sacrificed on day 19. Statistical analysis was performed using a two-way ANOVA test, followed by a Bonferroni post-test. B. Dex treatment did not affect in vivo KMS12-PE tumor growth. Mice were subcutaneously injected with $12 \times 10^{6} \mathrm{KMS} 12-\mathrm{PE}$ cells and treated as above. The mice were then sacrificed on day 17. Statistical analysis was performed using a two-way ANOVA test, followed by a Bonferroni post-test. 
homodimer binding and transactivation [32]. In the present study, we demonstrated that Dex induced an up-regulation of GILZ in all HMCLs but that this up-regulation was significantly higher in sensitive cell lines. Furthermore, we found a correlation between NR3C1 levels and TSC22D3 mRNA up-regulation. This result reinforces the fact that GILZ expression was proposed as a reliable measure of GR function [33]. We also demonstrated that GILZ silencing induced a strong reduction in Dex-induced cell death. This is in agreement with a study from Grugan et al [34], which has already implicated GILZ in Dex-induced apoptosis. However, in this previous study, the reduction in Dex-induced cell death caused by GILZ silencing was very modest $(10 \%)$. Altogether, these data now clearly indicate a pivotal role of GILZ in the induction of cell death by Dex. Although Dex-induced apoptosis required the contribution of multiple processes, modification of the Bcl-2 protein rheostat has been reported as a key process [35]. Particularly, BH3-only members have been shown to be important mediators of GC-induced apoptosis [14-16]. In our HMCL collection, we demonstrated that a strong Bim up-regulation associated with a weak Puma upregulation occurs mainly in Dex-sensitive cell lines, but also in the Dex-resistant KMS12-PE cell line. We further demonstrated that Bim silencing led to a strong reduction in apoptosis, which confirms the important role of Bim in the cell death process induced by Dex. In parallel with Bim up-regulation, we observed a strong down-regulation of Bcl- $\mathrm{x}_{\mathrm{L}}$, which is restricted to sensitive HMCLs. Thus, an imbalance between anti- and pro-apoptotic molecules occurred, leading to a decrease in the apoptotic threshold in Dex-sensitive cell lines. Of interest, GILZ silencing provided evidence that GILZ contributed to the regulation of the Bcl-2 protein network by inducing Bim up-regulation and $\mathrm{Bcl}-\mathrm{x}_{\mathrm{L}}$ down-regulation. The regulation of $\mathrm{Bcl}-\mathrm{x}_{\mathrm{L}}$ by GILZ is in agreement with a previous study showing that Bcl- $\mathrm{x}_{\mathrm{L}}$ is decreased in GILZ-overexpressing transgenic mice [36]. The implication of GILZ in Bim up-regulation remains an important question that deserves further investigation. Altogether, our data reinforce the importance of GILZ in the pro-apoptotic effect of GC in hematopoietic tumor cells.

Whereas CCND1 HMCLs have a low level of GR, which could in part account for their resistance to Dex, KMS12-PE cells exhibited a high level of GR, leading to both a significant induction of GILZ and up-regulation of Bim without the induction of apoptosis. Because Dex did not induced $\mathrm{Bcl}-\mathrm{x}_{\mathrm{L}}$ down-regulation in KMS12-PE cells as it did in other sensitive cell lines, we forced this down-regulation, showing that concomitant $\mathrm{Bcl}-\mathrm{x}_{\mathrm{L}}$ downregulation, Bim up-regulation and GILZ induction were not sufficient to counteract the Dex resistance of KMS12-PE cells. We cannot rule out other factors that may contribute to the Dex response. Finally, the in vivo growth of myeloma cells in our mouse model confirmed the heterogeneity of Dex efficacy, which corroborated our in vitro findings. Indeed, whereas Dex led to a reduction of MS tumor growth, Dex had no effect on CCND1 tumor growth. Of note, the dual action of GCs in survival has been reported $[37,38]$. Indeed, it was demonstrated that although Dex induced the apoptosis of a large majority of primary leukemia cells from children with newly diagnosed acute leukemia, the survival of a subset of primary acute leukemia samples (32\%) was increased by GCs [37]. Furthermore, our results seem to indicate that Dex had no pro-apoptotic effects on CCND1 myeloma cells both in vitro and in vivo, despite the presence of effective GR. Interestingly, the CCND1 subgroup has already been distinguished from other subgroups by its particular anti- and pro-apoptotic Bcl-2 member expression and its specific drug response [39, 40]. Altogether, these results indicated that both the MF and CCND1 subgroups presented important specificities that require better analysis during the course of the disease and the response to treatment. The risk/benefit of prolonged GC treatment should be re-evaluated to propose optimal treatments by GCs for each molecular group of MM.

\section{MATERIALS AND METHODS}

\section{Cell lines, primary myeloma cells and culture conditions}

HMCLs BCN, MDN, SBN, NAN-1, $-6,-7,-8,-10$ and $\mathrm{XG}-1,-2,-3,-5,-6,-7$ were derived in our laboratory from primary myeloma cells in the presence of interleukin (IL)-6. ANBL-6 was kindly provided by Dr. Jelinek (Rochester, MN). KMS11, KMS12-BM, KMS12-PE, and KMM1 were kindly provided by Dr. Otsuki (Kurashiki, Japan). JJN3, JIM3, Karpas620, and MM.1S were, respectively, kindly provided by Drs. Van Riet (Brussels, Belgium), MacLennan (Birmingham, UK), Karpas (Cambridge, UK), and S. Rosen (Chicago, IL). AMO1, LP1, L363, NCI-H929, SKMM2, U266, and OPM2 were purchased from the DSMZ, and RPMI8226 from American Type Culture Collection. Each HMCL was characterized and identified as previously described [41-43].

After obtaining informed consent, blood or bone marrow samples from MM patients were collected at the Department of Hematology at the University Hospital of Nantes or at the Intergroupe Francophone du Myélome (ethical approval n DC-2011-1399, Pr Rodat). Plasma cells were purified with CD138 immunomagnetic beads. The purity of the plasma cells was greater than $90 \%$, as assessed morphologically or by CD138 staining.

\section{Reagents}

Dexamethasone was purchased from SigmaAldrich (Saint-Quentin Fallavier, France) and dissolved in ethanol at $1 \mathrm{mM}$ as a stock solution. For the in vivo study, Dex was dissolved in ethanol and then in PBS at a final Dex concentration of $0.2 \mathrm{mg} / \mathrm{ml}$, with a final ethanol concentration of less than $3 \%$. 


\section{Cell death assays}

The evaluation of cell death in HMCLs was performed by flow cytometry using Apo2.7 staining. Cell death in primary myeloma cells was measured after 48 hours of culture in RPMI-1640 containing 5\% FCS with or without Dex $(1 \mu \mathrm{M})$ by assessing the loss of CD138 staining, as previously described [42].

\section{Establishment of Dex-resistant cell lines}

To establish Dex-resistant cell lines, NAN8 and MM.1S cells were continuously treated with Dex beginning with a dose of $0.001 \mu \mathrm{M}$. After cells displayed a viability of $80 \%$ under continuous exposure to a low Dex concentration, the drug dose was doubled. This process was repeated until cells were cultured in a Dex concentration of $10 \mu \mathrm{M}$. Prior to the experiments, the cells were cultured in Dex-free medium for 5 days before use.

\section{Immunoblotting}

Western blotting analyses were performed as previously described [44]. The following antibodies were used: caspase-3 (E-8), GR (E-20), lamin A/C (E-1), GILZ (FL-134), Mcl-1 (S-19) (Santa Cruz, Heidelberg, Germany), Puma (D30C10) (Cell Signaling Technology, Saint Quentin, France), tubulin (GE Healthcare Life Sciences, Velizy, France), Bcl- $\mathrm{x}_{\mathrm{L}}$ (BD Transduction Laboratories, Rungis, France), Bim, PARP1 (Ab-2) and actin (Merck Chemicals, Nottingham, UK). Protein expression was quantified and normalized to actin expression using ImageJ software.

\section{RNA interference assays}

Control nontargeted siRNA, siBim and siGILZ were purchased from Santa Cruz Biotechnology (Heidelberg, Germany), and siBcl- $\mathrm{x}_{\mathrm{L}}$ was purchased from Thermo Scientific (Ilkirch, France). OPM2 cells were transfected using Lipofectamine RNAiMAX (Invitrogen, Life Technologies, St Aubin, France) according to the manufacturer's instructions. Briefly, cells were plated at $1 \times 10^{6}$ cells per well in a 6-well plate. After 24 hours, 100 pmol siRNA was transfected into the cells using Lipofectamine RNAiMAX reagent. Cells were incubated for 72 hours for siBim and 48 hours for siGILZ before being subjected to various analyses. KMS12-PE and BCN cells were transfected using the Amaxa 4D-Nucleofector (Lonza, Cologne, Germany). The cell pellet was resuspended with $100 \mu \mathrm{l}$ solution F (Lonza) containing 100 pmol siRNA and electroporated using the CA137 or the DN100 program for KMS12-PE and BCN, respectively. After 24 hours, the cells were treated with Dex.

\section{Affymetrix gene expression}

Gene expression analyses of newly diagnosed MM patients were performed with the publicly available
Affymetrix Amazonia database (http://amazonia. transcriptome.eu/). HMCL gene expression analyses were performed with the RAGE database. The probes used for analyses were as follows: 211671_s_at for the NR3C1 gene, 209348_s_at for the $c M A F$ gene and 218559_s_at for the $M A F B$ gene.

\section{RNA extraction, reverse transcription and quantitative real-time PCR}

RNA extraction, reverse transcription and Q-PCR were performed as previously described [45]. TaqMan gene expression assays for $\mathrm{NR} 3 \mathrm{Cl}$ (Hs00353740_m1), TSC22D3 (Hs00608272_m1), BCL2L11 (Hs00708019_s1) and RPL37A (Hs01102345_m1, housekeeping gene) were obtained from Applied Biosystems (Life Technologies, St Aubin, France).

\section{Cell fractionation}

Cell fractionation was performed using a Nuclear Extraction kit (Cayman Chemical, Ann Arbor, USA) by following the manufacturer's protocol. Briefly, the cytosolic fraction was first obtained after lysis using a hypotonic buffer. Then, the nuclear fraction was obtained after 30 minutes incubation with a nuclear extraction buffer.

\section{Xenotransplant}

Female, beige, 6-week-old SCID mice were purchased from Charles River (L'Arbresle, France). Mice were bred and housed in the Experimental Therapeutic Unit (UTE, SFR Bonamy, Nantes, France) under animal care license $\mathrm{n}^{\circ} 44565$. OPM2 or KMS12-PE tumors were generated by implanting $12 \times 10^{6}$ cells in $100 \mu \mathrm{l}$ of PBS/Matrigel (1:1, Corning) in the right flank above the hind leg [46]. The tumor volume was recorded in three dimensions using a digital caliper and calculated as the length $\mathrm{x}$ width $\mathrm{x}$ depth. When the mice bore similar tumor sizes (approximately $100 \mathrm{~mm}^{3}$ ), they were randomly separated into 2 groups (6 mice per group) and received an intraperitoneal injection (i.p.) of either vehicle (PBS-diluted ethanol) or Dex (1 mg/ $\mathrm{kg}) 5$ days a week for 3 consecutive weeks. Animals were sacrificed when their tumor sizes reached $2000 \mathrm{~mm}^{3}$.

\section{Statistical analysis}

Statistical analyses were conducted using MannWhitney, Kruskal-Wallis, Spearman, Wilcoxon matchedpairs signed rank or a two-way ANOVA test.

\section{ACKNOWLEDGMENTS}

We thank Tumorothèque IRCNA (CHU and ICO, Nantes, France) for providing us with purified myeloma cells. 


\section{GRANT SUPPORT}

This work was supported by grants from Actions Cancer 44

\section{CONFLICTS OF INTEREST}

The authors indicate no potential conflict of interest.

\section{REFERENCES}

1. Kumar SK, Rajkumar SV, Dispenzieri A, Lacy MQ, Hayman SR, Buadi FK, Zeldenrust SR, Dingli D, Russell SJ, Lust JA, Greipp PR, Kyle RA, Gertz MA. Improved survival in multiple myeloma and the impact of novel therapies. Blood. 2008; 111:2516-2520.

2. Alexanian R, Dimopoulos MA, Delasalle K, Barlogie B. Primary dexamethasone treatment of multiple myeloma. Blood. 1992; 80:887-890.

3. Rajkumar SV, Blood E, Vesole D, Fonseca R, Greipp PR Eastern Cooperative Oncology G. Phase III clinical trial of thalidomide plus dexamethasone compared with dexamethasone alone in newly diagnosed multiple myeloma: a clinical trial coordinated by the Eastern Cooperative Oncology Group. Journal of clinical oncology: official journal of the American Society of Clinical Oncology. 2006; 24:431-436.

4. Chesi M, Bergsagel PL. Molecular pathogenesis of multiple myeloma: basic and clinical updates. International journal of hematology. 2013; 97:313-323.

5. Cruz-Topete D, Cidlowski JA. One hormone, two actions: anti- and pro-inflammatory effects of glucocorticoids. Neuroimmunomodulation. 2015; 22:20-32.

6. Smith LK, Cidlowski JA. Glucocorticoid-induced apoptosis of healthy and malignant lymphocytes. Progress in brain research. 2010; 182:1-30.

7. Gruver-Yates AL, Quinn MA, Cidlowski JA. Analysis of glucocorticoid receptors and their apoptotic response to dexamethasone in male murine B cells during development. Endocrinology. 2014; 155:463-474.

8. Gross KL, Lu NZ, Cidlowski JA. Molecular mechanisms regulating glucocorticoid sensitivity and resistance. Molecular and cellular endocrinology. 2009; 300:7-16.

9. Distelhorst CW. Recent insights into the mechanism of glucocorticosteroid-induced apoptosis. Cell death and differentiation. 2002; 9:6-19.

10. Rainer J, Lelong J, Bindreither D, Mantinger C, Ploner C, Geley S, Kofler R. Research resource: transcriptional response to glucocorticoids in childhood acute lymphoblastic leukemia. Molecular endocrinology. 2012; 26:178-193.

11. Ayroldi E, Riccardi C. Glucocorticoid-induced leucine zipper (GILZ): a new important mediator of glucocorticoid action. FASEB journal: official publication of the Federation of American Societies for Experimental Biology. 2009; 23:3649-3658.
12. Beaulieu E, Morand EF. Role of GILZ in immune regulation, glucocorticoid actions and rheumatoid arthritis. Nature reviews Rheumatology. 2011; 7:340-348.

13. Ayroldi E, Macchiarulo A, Riccardi C. Targeting glucocorticoid side effects: selective glucocorticoid receptor modulator or glucocorticoid-induced leucine zipper? A perspective: FASEB journal: official publication of the Federation of American Societies for Experimental Biology. 2014; 28:5055-5070.

14. Abrams MT, Robertson NM, Yoon K, Wickstrom E. Inhibition of glucocorticoid-induced apoptosis by targeting the major splice variants of BIM mRNA with small interfering RNA and short hairpin RNA. The Journal of biological chemistry. 2004; 279:55809-55817.

15. Erlacher M, Michalak EM, Kelly PN, Labi V, Niederegger H, Coultas L, Adams JM, Strasser A, Villunger A. BH3-only proteins Puma and Bim are rate-limiting for gamma-radiation- and glucocorticoidinduced apoptosis of lymphoid cells in vivo. Blood. 2005; 106:4131-4138.

16. Bachmann PS, Gorman R, Mackenzie KL, Lutze-Mann L, Lock RB. Dexamethasone resistance in B-cell precursor childhood acute lymphoblastic leukemia occurs downstream of ligand-induced nuclear translocation of the glucocorticoid receptor. Blood. 2005; 105:2519-2526.

17. Herve AL, Florence M, Philippe M, Michel A, Thierry F, Kenneth A, Jean-Luc H, Nikhil M, Stephane M. Molecular heterogeneity of multiple myeloma: pathogenesis, prognosis, and therapeutic implications. Journal of clinical oncology: official journal of the American Society of Clinical Oncology. 2011; 29:1893-1897.

18. Zhan F, Huang Y, Colla S, Stewart JP, Hanamura I, Gupta S, Epstein J, Yaccoby S, Sawyer J, Burington B, Anaissie E, Hollmig K, Pineda-Roman M, Tricot G, van Rhee F, Walker R, et al. The molecular classification of multiple myeloma. Blood. 2006; 108:2020-2028.

19. Abdi J, Chen G, Chang H. Drug resistance in multiple myeloma: latest findings and new concepts on molecular mechanisms. Oncotarget. 2013; 4:2186-2207.

20. Avet-Loiseau H, Attal M, Moreau P, Charbonnel C, Garban F, Hulin C, Leyvraz S, Michallet M, YakoubAgha I, Garderet L, Marit G, Michaux L, Voillat L, Renaud M, Grosbois B, Guillerm G, et al. Genetic abnormalities and survival in multiple myeloma: the experience of the Intergroupe Francophone du Myelome. Blood. 2007; 109:3489-3495.

21. Nair B, van Rhee F, Shaughnessy JD Jr, Anaissie E, Szymonifka J, Hoering A, Alsayed Y, Waheed S, Crowley J, Barlogie B. Superior results of Total Therapy 3 (2003-33) in gene expression profiling-defined low-risk multiple myeloma confirmed in subsequent trial 2006-66 with VRD maintenance. Blood. 2010; 115:4168-4173.

22. Hurt EM, Wiestner A, Rosenwald A, Shaffer AL, Campo E, Grogan T, Bergsagel PL, Kuehl WM, Staudt LM. Overexpression of c-maf is a frequent oncogenic event in 
multiple myeloma that promotes proliferation and pathological interactions with bone marrow stroma. Cancer cell. 2004; 5:191-199.

23. Annunziata CM, Hernandez L, Davis RE, Zingone A, Lamy L, Lam LT, Hurt EM, Shaffer AL, Kuehl WM, Staudt LM. A mechanistic rationale for MEK inhibitor therapy in myeloma based on blockade of MAF oncogene expression. Blood. 2011; 117:2396-2404.

24. Kumar S, Fonseca R, Ketterling RP, Dispenzieri A, Lacy MQ, Gertz MA, Hayman SR, Buadi FK, Dingli D, Knudson RA, Greenberg A, Russell SJ, Zeldenrust SR, Lust JA, Kyle RA, Bergsagel L, et al. Trisomies in multiple myeloma: impact on survival in patients with high-risk cytogenetics. Blood. 2012; 119:2100-2105.

25. Vandevyver S, Dejager L, Libert C. On the trail of the glucocorticoid receptor: into the nucleus and back. Traffic. 2012; 13:364-374.

26. Chauhan D, Auclair D, Robinson EK, Hideshima T, Li G, Podar K, Gupta D, Richardson P, Schlossman RL, Krett N, Chen LB, Munshi NC, Anderson KC. Identification of genes regulated by dexamethasone in multiple myeloma cells using oligonucleotide arrays. Oncogene. 2002; 21:1346-1358.

27. Mao X, Stewart AK, Hurren R, Datti A, Zhu X, Zhu Y, Shi C, Lee K, Tiedemann R, Eberhard Y, Trudel S, Liang S, Corey SJ, Gillis LC, Barber DL, Wrana JL, et al. A chemical biology screen identifies glucocorticoids that regulate c-maf expression by increasing its proteasomal degradation through up-regulation of ubiquitin. Blood. 2007; 110:4047-4054.

28. Murakami T, Togawa A, Satch H, Katoh M, Imamura Y, Ohsawa N, Takaku F. Glucocorticoid receptor in multiple myeloma. European journal of haematology. 1987; 39:54-59.

29. Heuck CJ, Szymonifka J, Hansen E, Shaughnessy JD Jr, Usmani SZ, van Rhee F, Anaissie E, Nair B, Waheed S, Alsayed Y, Petty N, Bailey C, Epstein J, Hoering A, Crowley J, Barlogie B. Thalidomide in total therapy 2 overcomes inferior prognosis of myeloma with low expression of the glucocorticoid receptor gene NR3C1. Clinical cancer research: an official journal of the American Association for Cancer Research. 2012; 18:5499-5506.

30. Ayroldi E, Migliorati G, Bruscoli S, Marchetti C, Zollo O, Cannarile L, D'Adamio F, Riccardi C. Modulation of T-cell activation by the glucocorticoid-induced leucine zipper factor via inhibition of nuclear factor kappaB. Blood. 2001; 98:743-753.

31. Cannarile L, Zollo O, D'Adamio F, Ayroldi E, Marchetti C, Tabilio A, Bruscoli S, Riccardi C. Cloning, chromosomal assignment and tissue distribution of human GILZ, a glucocorticoid hormone-induced gene. Cell death and differentiation. 2001; 8:201-203.

32. Asselin-Labat ML, David M, Biola-Vidamment A, Lecoeuche D, Zennaro MC, Bertoglio J, Pallardy M. GILZ, a new target for the transcription factor FoxO3, protects $\mathrm{T}$ lymphocytes from interleukin-2 withdrawal-induced apoptosis. Blood. 2004; 104:215-223.

33. Thiagarajah AS, Eades LE, Thomas PR, Guymer EK, Morand EF, Clarke DM, Leech M. GILZ: Glitzing up our understanding of the glucocorticoid receptor in psychopathology. Brain research. 2014; 1574:60-69.

34. Grugan KD, Ma C, Singhal S, Krett NL, Rosen ST. Dual regulation of glucocorticoid-induced leucine zipper (GILZ) by the glucocorticoid receptor and the PI3-kinase/AKT pathways in multiple myeloma. The Journal of steroid biochemistry and molecular biology. 2008; 110:244-254.

35. Ploner C, Rainer J, Niederegger H, Eduardoff $M$, Villunger A, Geley S, Kofler R. The BCL2 rheostat in glucocorticoid-induced apoptosis of acute lymphoblastic leukemia. Leukemia. 2008; 22:370-377.

36. Delfino DV, Agostini M, Spinicelli S, Vito P, Riccardi C. Decrease of Bcl-xL and augmentation of thymocyte apoptosis in GILZ overexpressing transgenic mice. Blood. 2004; 104:4134-4141.

37. Gundisch S, Boeckeler E, Behrends U, Amtmann E, Ehrhardt H, Jeremias I. Glucocorticoids augment survival and proliferation of tumor cells. Anticancer research. 2012; 32:4251-4261.

38. Al-Wadei HA, Takahasi T, Schuller HM. PKA-dependent growth stimulation of cells derived from human pulmonary adenocarcinoma and small airway epithelium by dexamethasone. European journal of cancer. 2005; 41:2745-2753.

39. Touzeau C, Dousset C, Le Gouill S, Sampath D, Leverson JD, Souers AJ, Maiga S, Bene MC, Moreau P, PellatDeceunynck $\mathrm{C}$, Amiot $\mathrm{M}$. The $\mathrm{Bcl}-2$ specific $\mathrm{BH} 3$ mimetic ABT-199: a promising targeted therapy for $\mathrm{t}(11 ; 14) \mathrm{mul}-$ tiple myeloma. Leukemia. 2014; 28:210-212.

40. Gomez-Bougie P, Amiot M. Apoptotic machinery diversity in multiple myeloma molecular subtypes. Frontiers in immunology. 2013; 4:467.

41. Moreaux J, Klein B, Bataille R, Descamps G, Maiga S, Hose D, Goldschmidt H, Jauch A, Reme T, Jourdan M, Amiot M, Pellat-Deceunynck C. A high-risk signature for patients with multiple myeloma established from the molecular classification of human myeloma cell lines. Haematologica. 2011; 96:574-582.

42. Surget S, Chiron D, Gomez-Bougie P, Descamps G, Menoret E, Bataille R, Moreau P, Le Gouill S, Amiot M, Pellat-Deceunynck C. Cell death via DR5, but not DR4, is regulated by p53 in myeloma cells. Cancer research. 2012; 72:4562-4573.

43. Maiga S, Brosseau C, Descamps G, Dousset C, Gomez-Bougie P, Chiron D, Menoret E, Kervoelen C, Vie H, Cesbron A, Moreau-Aubry A, Amiot M, PellatDeceunynck C. A simple flow cytometry-based barcode for routine authentication of multiple myeloma and mantle cell lymphoma cell lines. Cytometry Part A: the journal of the International Society for Analytical Cytology. 2015. 
44. Gomez-Bougie P, Bataille R, Amiot M. The imbalance between Bim and Mcl-1 expression controls the survival of human myeloma cells. European journal of immunology. 2004; 34:3156-3164.

45. Maiga S, Gomez-Bougie P, Bonnaud S, Gratas C, Moreau P, Le Gouill S, Pellat-Deceunynck C, Amiot M. Paradoxical effect of lenalidomide on cytokine/growth factor profiles in multiple myeloma. British journal of cancer. 2013; 108:1801-1806.

46. Tessoulin B, Descamps G, Moreau P, Maiga S, Lode L, Godon C, Marionneau-Lambot S, Oullier T, Le Gouill S, Amiot M, Pellat-Deceunynck C. PRIMA-1Met induces myeloma cell death independent of p53 by impairing the GSH/ROS balance. Blood. 2014; 124:1626-1636. 